Supporting Information for:

\title{
Viscosity and Density Measurements on Liquid n-heptadecane at High Pressures
}

Maria C. M. Sequeira ${ }^{1}$, Helena M. N. T. Avelino ${ }^{1,2, *}$, Fernando J. P. Caetano ${ }^{1,3}$, João M. N. A. Fareleira ${ }^{1, *}$

Corresponding Author:

htavelino@deq.isel.ipl.pt j.fareleira@tecnico.ulisboa.pt

\section{Annex A1}

The statistical parameters used to characterize the quality of the fittings in the present work are defined by Eqs. (1) and (2), namely, the relative root mean square deviation, hereby designated as $\sigma$ and the bias.

$$
\begin{aligned}
& \sigma=\left[\frac{1}{N} \sum_{\mathrm{i}}^{N}\left(\frac{X_{\text {exp,i }}}{X_{\text {calc, } \mathrm{i}}}-1\right)^{2}\right]^{1 / 2} \\
& \text { bias }=\frac{1}{N} \sum_{\mathrm{i}}^{N}\left(\frac{X_{\text {exp }, \mathrm{i}}}{X_{\text {calc,i }}}-1\right)
\end{aligned}
$$

where $N$ is the total number of experimental data points, the subscripts (exp,i and calc,i) stand for the ith experimental and calculated data points, respectively, and $X$ stands either for the density or the viscosity. 


\section{Table S1}

Density, $\rho$, data for $\mathrm{C}_{17}$ obtained with an Anton Paar DMA HP densimeter, at temperatures, $T$, from (303.15 to 358.15$) \mathrm{K}$ and pressures, $p$, up to $70 \mathrm{MPa}$

\begin{tabular}{|c|c|c|c|c|c|c|c|c|}
\hline$T / \mathrm{K}$ & $p / \mathrm{MPa}$ & $\rho /\left(\mathrm{kg} \cdot \mathrm{m}^{-3}\right)$ & $T / \mathrm{K}$ & $p / \mathrm{MPa}$ & $\rho /\left(\mathrm{kg} \cdot \mathrm{m}^{-3}\right)$ & $T / \mathrm{K}$ & $p / \mathrm{MPa}$ & $\rho /\left(\mathrm{kg} \cdot \mathrm{m}^{-3}\right)$ \\
\hline \multirow[t]{12}{*}{303.15} & 0.10 & 771.9 & 323.15 & 7.01 & 763.1 & 343.15 & 1.03 & 745.3 \\
\hline & 0.10 & 771.9 & & 9.88 & 765.1 & & 1.54 & 745.7 \\
\hline & 0.60 & 772.2 & & 14.92 & 768.3 & & 2.10 & 746.2 \\
\hline & 1.10 & 772.5 & & 19.71 & 771.4 & & 3.08 & 746.9 \\
\hline & 1.60 & 772.9 & & 24.70 & 774.4 & & 5.02 & 748.4 \\
\hline & 2.12 & 773.2 & & 29.58 & 777.4 & & 7.06 & 749.9 \\
\hline & 3.10 & 773.8 & & 34.49 & 780.3 & & 9.91 & 752.1 \\
\hline & 5.11 & 775.1 & & 39.35 & 782.9 & & 14.89 & 755.6 \\
\hline & 6.97 & 776.3 & & 49.20 & 788.3 & & 19.79 & 759.0 \\
\hline & 9.93 & 778.2 & & 54.08 & 790.8 & & 24.70 & 762.2 \\
\hline & 14.90 & 781.2 & & 58.92 & 792.9 & & 29.51 & 765.3 \\
\hline & 19.81 & 784.0 & & 63.89 & 795.5 & & 34.49 & 768.4 \\
\hline \multirow[t]{17}{*}{313.15} & 0.10 & 765.1 & & 68.81 & 797.7 & & 39.40 & 771.4 \\
\hline & 0.10 & 765.1 & 333.15 & 0.10 & 751.4 & & 44.29 & 774.2 \\
\hline & 0.68 & 765.5 & & 0.10 & 751.4 & & 54.09 & 779.8 \\
\hline & 1.15 & 765.8 & & 0.67 & 751.8 & & 59.01 & 782.3 \\
\hline & 1.64 & 766.1 & & 1.14 & 752.2 & & 63.90 & 784.7 \\
\hline & 2.10 & 766.5 & & 1.53 & 752.5 & & 68.85 & 787.3 \\
\hline & 3.02 & 767.1 & & 2.12 & 752.9 & & 0.10 & 734.3 \\
\hline & 5.11 & 768.5 & & 3.06 & 753.6 & & 0.10 & 734.2 \\
\hline & 7.06 & 769.8 & & 5.09 & 755.1 & & 0.66 & 734.7 \\
\hline & 9.99 & 771.7 & & 6.97 & 756.5 & 358.15 & 1.16 & 735.1 \\
\hline & 14.82 & 774.7 & & 9.98 & 758.6 & & 1.58 & 735.5 \\
\hline & 19.71 & 777.7 & & 14.85 & 761.9 & & 2.10 & 735.9 \\
\hline & 24.68 & 780.6 & & 19.75 & 765.2 & & 3.00 & 736.6 \\
\hline & 29.62 & 783.4 & & 24.72 & 768.3 & & 5.04 & 738.4 \\
\hline & 34.51 & 786.2 & & 29.53 & 771.3 & & 7.01 & 739.9 \\
\hline & 39.36 & 788.8 & & 34.47 & 774.2 & & 9.95 & 742.3 \\
\hline & 44.27 & 791.7 & & 44.27 & 780.1 & & 14.82 & 746.0 \\
\hline \multirow[t]{8}{*}{323.15} & 0.10 & 758.3 & & 49.24 & 782.5 & & 19.77 & 749.7 \\
\hline & 0.10 & 758.3 & & 54.09 & 785.1 & & 24.60 & 753.0 \\
\hline & 0.64 & 758.7 & & 58.97 & 787.6 & & 29.63 & 756.3 \\
\hline & 1.16 & 759.0 & & 63.84 & 790.1 & & 34.51 & 759.8 \\
\hline & 1.53 & 759.3 & & 68.77 & 792.6 & & 44.27 & 765.9 \\
\hline & 2.12 & 759.7 & 343.15 & 0.10 & 744.6 & & 59.00 & 774.1 \\
\hline & 3.07 & 760.3 & & 0.10 & 744.6 & & 63.89 & 776.8 \\
\hline & 5.08 & 761.8 & & 0.64 & 745.0 & & 68.78 & 779.4 \\
\hline
\end{tabular}


The combined expanded uncertainties $U(\mathrm{k}=2)$ are as follows: $U(T)=0.05 \mathrm{~K} ; U(p)=0.08 \mathrm{MPa} ; U(\rho)$ $=0.002 \cdot \rho$

\section{Table S2}

Viscosity, $\eta$, data for $\mathrm{C}_{17}$ measured with the vibrating wire technique, at temperatures, $T$, from (303.26 to 358.19) $\mathrm{K}$ and pressures, $p$, up to $70 \mathrm{MPa}$.

\begin{tabular}{|c|c|c|c|c|c|c|c|c|}
\hline$T / \mathrm{K}$ & $p / \mathrm{MPa}$ & $\eta /(\mathrm{mPa} \cdot \mathrm{s})$ & $T / \mathrm{K}$ & $p / \mathrm{MPa}$ & $\eta /(\mathrm{mPa} \cdot \mathrm{s})$ & $T / \mathrm{K}$ & $p / \mathrm{MPa}$ & $\eta /(\mathrm{mPa} \cdot \mathrm{s})$ \\
\hline \multirow[t]{38}{*}{303.26} & 0.19 & 3.219 & 323.21 & 5.12 & 2.252 & 343.16 & 1.12 & 1.523 \\
\hline & 0.19 & 3.204 & & 5.12 & 2.245 & & 1.11 & 1.526 \\
\hline & 0.19 & 3.214 & & 5.12 & 2.253 & & 2.14 & 1.544 \\
\hline & 0.19 & 3.199 & & 5.12 & 2.236 & & 2.14 & 1.537 \\
\hline & 0.20 & 3.212 & & 5.44 & 2.265 & & 2.14 & 1.551 \\
\hline & 0.20 & 3.217 & & 5.44 & 2.255 & & 2.14 & 1.537 \\
\hline & 0.20 & 3.221 & & 5.44 & 2.255 & & 2.14 & 1.542 \\
\hline & 0.20 & 3.217 & & 5.44 & 2.273 & & 2.14 & 1.548 \\
\hline & 0.20 & 3.212 & & 10.16 & 2.401 & & 2.15 & 1.534 \\
\hline & 0.20 & 3.207 & & 10.16 & 2.402 & & 2.15 & 1.536 \\
\hline & 1.26 & 3.239 & & 10.16 & 2.387 & & 2.15 & 1.545 \\
\hline & 1.26 & 3.257 & & 10.17 & 2.385 & & 2.15 & 1.546 \\
\hline & 1.26 & 3.255 & & 10.16 & 2.400 & & 5.12 & 1.597 \\
\hline & 1.26 & 3.259 & & 10.16 & 2.390 & & 5.12 & 1.597 \\
\hline & 1.26 & 3.241 & & 10.17 & 2.395 & & 5.12 & 1.599 \\
\hline & 1.26 & 3.236 & & 10.17 & 2.398 & & 5.12 & 1.605 \\
\hline & 1.26 & 3.246 & & 10.17 & 2.397 & & 5.12 & 1.597 \\
\hline & 1.26 & 3.247 & & 10.17 & 2.389 & & 5.12 & 1.599 \\
\hline & 1.26 & 3.258 & & 20.35 & 2.718 & & 5.12 & 1.595 \\
\hline & 1.26 & 3.234 & & 20.35 & 2.708 & & 5.12 & 1.602 \\
\hline & 2.14 & 3.307 & & 20.35 & 2.714 & & 5.12 & 1.591 \\
\hline & 2.14 & 3.300 & & 20.35 & 2.702 & & 5.44 & 1.610 \\
\hline & 2.14 & 3.274 & & 20.35 & 2.728 & & 5.44 & 1.610 \\
\hline & 2.14 & 3.280 & & 20.35 & 2.709 & & 5.44 & 1.607 \\
\hline & 2.14 & 3.304 & & 20.35 & 2.709 & & 5.44 & 1.609 \\
\hline & 2.14 & 3.302 & & 20.35 & 2.708 & & 5.44 & 1.616 \\
\hline & 2.13 & 3.290 & & 20.35 & 2.725 & & 5.44 & 1.612 \\
\hline & 2.14 & 3.284 & & 20.35 & 2.718 & & 10.12 & 1.698 \\
\hline & 2.13 & 3.281 & & 30.02 & 3.028 & & 10.12 & 1.694 \\
\hline & 5.27 & 3.424 & & 30.02 & 3.031 & & 10.12 & 1.695 \\
\hline & 5.27 & 3.432 & & 30.02 & 3.010 & & 10.12 & 1.699 \\
\hline & 5.27 & 3.399 & & 30.02 & 3.021 & & 10.12 & 1.713 \\
\hline & 5.27 & 3.439 & & 30.02 & 3.055 & & 10.12 & 1.695 \\
\hline & 5.27 & 3.417 & & 30.02 & 3.047 & & 10.12 & 1.700 \\
\hline & 5.27 & 3.416 & & 30.02 & 3.027 & & 10.12 & 1.689 \\
\hline & 5.27 & 3.423 & & 30.01 & 3.044 & & 10.12 & 1.705 \\
\hline & 5.27 & 3.439 & & 30.01 & 3.029 & & 20.40 & 1.917 \\
\hline & 5.27 & 3.428 & & 30.01 & 3.034 & & 20.40 & 1.919 \\
\hline
\end{tabular}




\begin{tabular}{|c|c|c|c|c|c|c|c|}
\hline & 5.27 & 3.422 & & 40.20 & 3.419 & 20.40 & 1.906 \\
\hline & 5.27 & 3.449 & & 40.20 & 3.430 & 20.40 & 1.906 \\
\hline & 5.26 & 3.447 & & 40.19 & 3.416 & 20.40 & 1.915 \\
\hline & 5.26 & 3.428 & & 40.19 & 3.426 & 20.40 & 1.922 \\
\hline & 5.26 & 3.397 & & 40.19 & 3.416 & 20.40 & 1.904 \\
\hline & 5.27 & 3.445 & & 40.18 & 3.415 & 30.21 & 2.113 \\
\hline & 5.27 & 3.431 & & 40.18 & 3.405 & 30.21 & 2.134 \\
\hline & 10.29 & 3.657 & & 40.18 & 3.409 & 30.21 & 2.139 \\
\hline & 10.29 & 3.630 & & 40.18 & 3.404 & 30.21 & 2.127 \\
\hline & 10.29 & 3.635 & & 49.98 & 3.754 & 30.21 & 2.127 \\
\hline & 10.29 & 3.645 & & 49.98 & 3.778 & 30.21 & 2.133 \\
\hline & 10.29 & 3.654 & & 49.98 & 3.759 & 30.21 & 2.139 \\
\hline & 10.29 & 3.648 & & 49.97 & 3.767 & 30.21 & 2.130 \\
\hline & 10.29 & 3.648 & & 49.97 & 3.762 & 30.21 & 2.129 \\
\hline & 10.29 & 3.651 & & 49.96 & 3.785 & 39.84 & 2.336 \\
\hline & 10.29 & 3.652 & & 49.96 & 3.780 & 39.83 & 2.370 \\
\hline & 20.17 & 4.120 & & 49.96 & 3.760 & 39.83 & 2.364 \\
\hline & 20.17 & 4.136 & & 49.96 & 3.778 & 39.83 & 2.374 \\
\hline & 20.17 & 4.117 & & 49.96 & 3.787 & 39.82 & 2.338 \\
\hline & 20.17 & 4.156 & & 60.19 & 4.219 & 39.82 & 2.359 \\
\hline & 20.16 & 4.118 & & 60.16 & 4.205 & 39.82 & 2.350 \\
\hline & 20.16 & 4.137 & & 60.11 & 4.175 & 39.82 & 2.365 \\
\hline & 20.16 & 4.132 & & 60.08 & 4.193 & 39.82 & 2.367 \\
\hline & 20.16 & 4.153 & & 60.05 & 4.190 & 50.17 & 2.609 \\
\hline & 20.16 & 4.168 & & 60.03 & 4.193 & 50.17 & 2.633 \\
\hline 313.25 & 0.13 & 2.601 & & 60.00 & 4.210 & 50.16 & 2.613 \\
\hline & 0.14 & 2.594 & & 59.97 & 4.161 & 50.16 & 2.615 \\
\hline & 0.14 & 2.587 & & 59.94 & 4.170 & 50.15 & 2.622 \\
\hline & 0.14 & 2.586 & 333.19 & 0.22 & 1.767 & 50.15 & 2.630 \\
\hline & 0.14 & 2.601 & & 0.22 & 1.776 & 50.14 & 2.631 \\
\hline & 0.14 & 2.573 & & 0.22 & 1.773 & 50.14 & 2.609 \\
\hline & 0.14 & 2.579 & & 0.22 & 1.775 & 50.13 & 2.623 \\
\hline & 0.14 & 2.597 & & 0.22 & 1.779 & 50.13 & 2.620 \\
\hline & 0.14 & 2.587 & & 0.22 & 1.775 & 60.14 & 2.878 \\
\hline & 0.14 & 2.590 & & 0.22 & 1.785 & 60.12 & 2.873 \\
\hline & 1.26 & 2.612 & & 0.22 & 1.779 & 60.10 & 2.882 \\
\hline & 1.26 & 2.610 & & 0.22 & 1.775 & 60.06 & 2.885 \\
\hline & 1.26 & 2.611 & & 1.14 & 1.787 & 60.05 & 2.867 \\
\hline & 1.26 & 2.603 & & 1.15 & 1.790 & 60.02 & 2.863 \\
\hline & 1.26 & 2.629 & & 1.15 & 1.783 & 60.00 & 2.897 \\
\hline & 1.26 & 2.607 & & 1.15 & 1.793 & 69.03 & 3.155 \\
\hline & 1.26 & 2.609 & & 1.15 & 1.791 & 68.95 & 3.130 \\
\hline & 1.26 & 2.617 & & 1.15 & 1.782 & 68.88 & 3.124 \\
\hline & 1.26 & 2.625 & & 1.15 & 1.793 & 68.80 & 3.130 \\
\hline & 1.18 & 2.631 & & 1.15 & 1.787 & 68.71 & 3.134 \\
\hline & 1.18 & 2.632 & & 2.32 & 1.803 & 68.63 & 3.135 \\
\hline & 1.18 & 2.634 & & 2.32 & 1.808 & 68.56 & 3.110 \\
\hline & 1.18 & 2.619 & & 2.32 & 1.809 & 68.48 & 3.144 \\
\hline & 1.19 & 2.628 & & 2.32 & 1.812 & 68.41 & 3.122 \\
\hline
\end{tabular}




\begin{tabular}{|c|c|c|c|c|c|c|}
\hline 1.19 & 2.622 & 2.32 & 1.812 & & 68.33 & 3.114 \\
\hline 2.14 & 2.646 & 2.32 & 1.808 & 358.19 & 0.23 & 1.210 \\
\hline 2.14 & 2.644 & 2.32 & 1.813 & & 0.22 & 1.215 \\
\hline 2.14 & 2.651 & 2.32 & 1.809 & & 0.23 & 1.208 \\
\hline 2.14 & 2.652 & 2.32 & 1.806 & & 0.23 & 1.221 \\
\hline 2.14 & 2.645 & 2.32 & 1.805 & & 0.23 & 1.215 \\
\hline 2.14 & 2.644 & 5.09 & 1.885 & & 0.22 & 1.202 \\
\hline 2.14 & 2.646 & 5.09 & 1.877 & & 0.22 & 1.210 \\
\hline 2.14 & 2.646 & 5.09 & 1.881 & & 0.22 & 1.213 \\
\hline 2.14 & 2.644 & 5.09 & 1.894 & & 0.22 & 1.217 \\
\hline 5.34 & 2.742 & 5.09 & 1.875 & & 1.15 & 1.225 \\
\hline 5.34 & 2.760 & 5.09 & 1.882 & & 1.15 & 1.222 \\
\hline 5.34 & 2.766 & 5.09 & 1.889 & & 1.15 & 1.223 \\
\hline 5.34 & 2.762 & 5.09 & 1.867 & & 1.15 & 1.230 \\
\hline 5.34 & 2.761 & 5.09 & 1.877 & & 1.15 & 1.229 \\
\hline 5.34 & 2.757 & 5.09 & 1.886 & & 1.15 & 1.237 \\
\hline 5.34 & 2.746 & 5.20 & 1.887 & & 1.15 & 1.232 \\
\hline 5.34 & 2.748 & 5.20 & 1.879 & & 1.15 & 1.231 \\
\hline 5.34 & 2.759 & 5.20 & 1.882 & & 2.04 & 1.237 \\
\hline 5.34 & 2.757 & 5.20 & 1.880 & & 2.05 & 1.233 \\
\hline 11.03 & 2.958 & 5.20 & 1.889 & & 2.04 & 1.235 \\
\hline 11.03 & 2.969 & 5.20 & 1.879 & & 2.04 & 1.235 \\
\hline 11.03 & 2.971 & 10.23 & 1.996 & & 2.05 & 1.231 \\
\hline 11.03 & 2.948 & 10.23 & 1.998 & & 2.06 & 1.237 \\
\hline 11.03 & 2.966 & 10.23 & 2.005 & & 2.06 & 1.239 \\
\hline 11.03 & 2.959 & 10.23 & 1.990 & & 2.06 & 1.244 \\
\hline 11.03 & 2.978 & 10.23 & 1.987 & & 5.12 & 1.283 \\
\hline 11.03 & 2.969 & 10.23 & 1.986 & & 5.12 & 1.278 \\
\hline 11.03 & 2.977 & 10.23 & 1.997 & & 5.12 & 1.279 \\
\hline 11.03 & 2.975 & 10.23 & 1.995 & & 5.12 & 1.285 \\
\hline 20.20 & 3.339 & 10.23 & 2.003 & & 5.12 & 1.281 \\
\hline 20.20 & 3.339 & 10.23 & 1.991 & & 5.12 & 1.278 \\
\hline 20.20 & 3.331 & 20.08 & 2.263 & & 5.12 & 1.281 \\
\hline 20.20 & 3.326 & 20.08 & 2.262 & & 5.12 & 1.278 \\
\hline 20.20 & 3.334 & 20.08 & 2.238 & & 5.12 & 1.281 \\
\hline 20.20 & 3.322 & 20.08 & 2.254 & & 5.21 & 1.283 \\
\hline 20.20 & 3.336 & 20.08 & 2.252 & & 5.21 & 1.276 \\
\hline 20.20 & 3.326 & 20.08 & 2.250 & & 5.21 & 1.286 \\
\hline 20.19 & 3.323 & 20.07 & 2.266 & & 5.21 & 1.286 \\
\hline 30.82 & 3.781 & 30.22 & 2.525 & & 5.22 & 1.287 \\
\hline 30.82 & 3.783 & 30.22 & 2.525 & & 5.21 & 1.288 \\
\hline 30.82 & 3.776 & 30.22 & 2.527 & & 10.13 & 1.365 \\
\hline 30.82 & 3.775 & 30.22 & 2.528 & & 10.13 & 1.366 \\
\hline 30.82 & 3.768 & 30.22 & 2.524 & & 10.13 & 1.354 \\
\hline 30.82 & 3.771 & 30.21 & 2.531 & & 10.13 & 1.370 \\
\hline 30.82 & 3.760 & 30.21 & 2.530 & & 10.13 & 1.364 \\
\hline 30.81 & 3.782 & 30.21 & 2.533 & & 10.13 & 1.364 \\
\hline 30.81 & 3.778 & 30.21 & 2.525 & & 10.13 & 1.359 \\
\hline 39.86 & 4.144 & 30.21 & 2.519 & & 10.13 & 1.359 \\
\hline
\end{tabular}




\begin{tabular}{|c|c|c|c|c|c|c|c|}
\hline & 39.88 & 4.196 & & 40.09 & 2.812 & 10.14 & 1.359 \\
\hline & 39.87 & 4.169 & & 40.08 & 2.822 & 10.13 & 1.370 \\
\hline & 39.86 & 4.183 & & 40.08 & 2.819 & 20.06 & 1.514 \\
\hline & 39.85 & 4.166 & & 40.08 & 2.815 & 20.06 & 1.516 \\
\hline & 39.85 & 4.190 & & 40.08 & 2.801 & 20.06 & 1.518 \\
\hline & 39.85 & 4.172 & & 40.08 & 2.828 & 20.06 & 1.520 \\
\hline & 40.51 & 4.226 & & 40.08 & 2.810 & 20.06 & 1.514 \\
\hline & 40.50 & 4.214 & & 40.08 & 2.799 & 20.06 & 1.514 \\
\hline & 40.49 & 4.222 & & 40.07 & 2.793 & 20.06 & 1.511 \\
\hline & 40.49 & 4.213 & & 40.07 & 2.829 & 20.06 & 1.515 \\
\hline & 40.48 & 4.225 & & 50.08 & 3.121 & 20.06 & 1.513 \\
\hline & 40.46 & 4.220 & & 50.07 & 3.093 & 20.06 & 1.521 \\
\hline & 40.45 & 4.208 & & 50.06 & 3.146 & 30.12 & 1.700 \\
\hline & 40.45 & 4.208 & & 50.06 & 3.105 & 30.12 & 1.682 \\
\hline & 49.75 & 4.676 & & 50.05 & 3.132 & 30.11 & 1.697 \\
\hline & 49.75 & 4.669 & & 50.05 & 3.112 & 30.11 & 1.691 \\
\hline & 49.74 & 4.690 & & 50.04 & 3.130 & 30.11 & 1.690 \\
\hline & 49.74 & 4.667 & & 50.04 & 3.103 & 30.11 & 1.692 \\
\hline & 49.73 & 4.673 & & 50.03 & 3.126 & 30.11 & 1.697 \\
\hline & 49.72 & 4.680 & & 50.03 & 3.121 & 30.11 & 1.705 \\
\hline & 49.71 & 4.673 & & 59.88 & 3.447 & 30.11 & 1.697 \\
\hline & 49.70 & 4.665 & & 59.86 & 3.410 & 30.11 & 1.680 \\
\hline 323.22 & 0.15 & 2.114 & & 59.85 & 3.432 & 39.88 & 1.871 \\
\hline & 0.15 & 2.114 & & 59.84 & 3.444 & 39.87 & 1.879 \\
\hline & 0.15 & 2.115 & & 59.82 & 3.443 & 39.87 & 1.891 \\
\hline & 0.15 & 2.117 & & 59.81 & 3.436 & 39.87 & 1.883 \\
\hline & 0.15 & 2.121 & & 59.79 & 3.432 & 39.87 & 1.866 \\
\hline & 0.15 & 2.120 & & 59.78 & 3.442 & 39.86 & 1.873 \\
\hline & 0.15 & 2.108 & & 59.76 & 3.459 & 39.86 & 1.879 \\
\hline & 0.15 & 2.108 & & 59.74 & 3.425 & 39.86 & 1.871 \\
\hline & 0.15 & 2.125 & & 68.25 & 3.740 & 39.86 & 1.873 \\
\hline & 0.15 & 2.117 & & 68.25 & 3.702 & 50.12 & 2.073 \\
\hline & 1.17 & 2.142 & & 68.03 & 3.704 & 50.11 & 2.081 \\
\hline & 1.17 & 2.149 & & 67.92 & 3.715 & 50.11 & 2.086 \\
\hline & 1.17 & 2.146 & & 67.83 & 3.692 & 50.10 & 2.078 \\
\hline & 1.17 & 2.136 & & 67.73 & 3.717 & 50.10 & 2.082 \\
\hline & 1.17 & 2.149 & & 67.64 & 3.711 & 50.10 & 2.073 \\
\hline & 1.17 & 2.136 & & 67.55 & 3.678 & 50.09 & 2.079 \\
\hline & 1.17 & 2.144 & & 67.46 & 3.711 & 50.08 & 2.084 \\
\hline & 1.17 & 2.160 & & 67.37 & 3.683 & 50.07 & 2.077 \\
\hline & 1.17 & 2.135 & 343.16 & 0.20 & 1.511 & 59.93 & 2.291 \\
\hline & 1.17 & 2.137 & & 0.20 & 1.513 & 59.92 & 2.292 \\
\hline & 2.30 & 2.168 & & 0.20 & 1.501 & 59.91 & 2.286 \\
\hline & 2.30 & 2.171 & & 0.20 & 1.501 & 59.90 & 2.288 \\
\hline & 2.30 & 2.169 & & 0.20 & 1.498 & 59.89 & 2.298 \\
\hline & 2.31 & 2.171 & & 0.20 & 1.514 & 59.88 & 2.292 \\
\hline & 2.31 & 2.182 & & 0.20 & 1.500 & 59.87 & 2.281 \\
\hline & 2.31 & 2.172 & & 0.20 & 1.507 & 59.86 & 2.277 \\
\hline & 2.31 & 2.175 & & 0.19 & 1.514 & 59.85 & 2.293 \\
\hline
\end{tabular}




\begin{tabular}{llllll}
2.31 & 2.173 & 0.19 & 1.507 & 59.84 & 2.273 \\
2.31 & 2.182 & 1.12 & 1.521 & 69.14 & 2.474 \\
2.31 & 2.178 & 1.12 & 1.527 & 69.10 & 2.477 \\
5.12 & 2.254 & 1.12 & 1.523 & 69.07 & 2.476 \\
5.12 & 2.238 & 1.12 & 1.516 & 69.04 & 2.489 \\
5.12 & 2.253 & 1.12 & 1.521 & 68.97 & 2.470 \\
5.12 & 2.245 & 1.12 & 1.519 & 68.93 & 2.467 \\
5.12 & 2.248 & 1.12 & 1.526 & 68.87 & 2.468 \\
5.11 & 2.257 & 1.12 & 1.516 & 68.83 & 2.484 \\
\hline \hline
\end{tabular}

The combined expanded uncertainties $U(\mathrm{k}=2)$ are as follows: $U(T)=0.05 \mathrm{~K} ; U(p)=0.08 \mathrm{MPa} ; U(\eta)$ $=0.015 \cdot \eta$

Table S3.

Isobaric thermal expansivity, $\alpha_{\mathrm{p}}$, and isothermal compressibility, $\kappa_{\mathrm{T}}$, for $\mathrm{n}-\mathrm{C} 17$ at temperatures, $T$, from (303.15 to 358.15 ) $\mathrm{K}$ and pressures, $p$, up to $70 \mathrm{MPa}$

\begin{tabular}{cccccccc}
\hline \hline$T / \mathrm{K}$ & $p / \mathrm{MPa}$ & $10^{4} \mathrm{k}_{\mathrm{T}} / \mathrm{MPa}^{-1}$ & $10^{4} \alpha_{\mathrm{p}} / \mathrm{K}^{-1}$ & $T / \mathrm{K}$ & $p / \mathrm{MPa}$ & $10^{4} \mathrm{k}_{\mathrm{T}} / \mathrm{MPa}^{-1}$ & $10^{4} \alpha_{\mathrm{p}} / \mathrm{K}^{-1}$ \\
\hline 303.15 & 0.1 & 8.71 & 8.73 & 333.15 & 20 & 8.49 & 8.01 \\
& 1 & 8.64 & 8.69 & & 30 & 7.85 & 7.64 \\
& 5 & 8.36 & 8.54 & & 40 & 7.30 & 7.32 \\
& 10 & 8.03 & 8.36 & & 50 & 6.82 & 7.04 \\
& 20 & 7.44 & 8.04 & & 60 & 6.41 & 6.79 \\
313.15 & 0.1 & 9.15 & 8.81 & & 70 & 6.04 & 6.58 \\
& 1 & 9.07 & 8.76 & 343.15 & 0.1 & 10.79 & 9.04 \\
& 5 & 8.76 & 8.59 & & 1 & 10.69 & 8.99 \\
& 10 & 8.39 & 8.38 & & 5 & 10.25 & 8.75 \\
& 20 & 7.76 & 8.02 & & 10 & 9.76 & 8.48 \\
& 30 & 7.21 & 7.71 & & 20 & 8.91 & 8.02 \\
& 40 & 6.74 & 7.44 & & 30 & 8.20 & 7.62 \\
& 0.1 & 9.64 & 8.88 & & 40 & 7.60 & 7.29 \\
& 1 & 9.56 & 8.84 & & 50 & 7.09 & 6.99 \\
& 5 & 9.21 & 8.64 & & 60 & 6.65 & 6.74 \\
& 10 & 8.81 & 8.41 & & 70 & 6.26 & 6.51 \\
& 20 & 8.11 & 8.01 & 358.15 & 0.1 & 11.77 & 9.17 \\
& 30 & 7.52 & 7.67 & & 1 & 11.65 & 9.11 \\
& 40 & 7.01 & 7.37 & & 5 & 11.13 & 8.85 \\
& 50 & 6.57 & 7.11 & & 10 & 10.55 & 8.57 \\
& 60 & 6.18 & 6.88 & & 20 & 9.57 & 8.07 \\
& 70 & 5.84 & 6.68 & & 30 & 8.76 & 7.66 \\
& 0.1 & 10.19 & 8.96 & & 40 & 8.08 & 7.32 \\
& 1 & 10.10 & 8.91 & & 50 & 7.51 & 7.02 \\
& 5 & 9.71 & 8.69 & & 60 & 7.01 & 6.76 \\
& 10 & 9.26 & 8.44 & & 70 & 6.58 & 6.53 \\
\hline
\end{tabular}




\section{Table S4}

Pressure-viscosity coefficients, $\alpha$, for $\mathrm{n}-\mathrm{C} 17$, at temperatures, $T$, from (303 to 358$) \mathrm{K}$ and pressures, $p$, up to $70 \mathrm{MPa}$

\begin{tabular}{cccccc}
\hline \hline$T / \mathrm{K}$ & $p / \mathrm{Mpa}$ & $\alpha / \mathrm{GPa}^{-1}$ & $T / \mathrm{K}$ & $p / \mathrm{Mpa}$ & $\alpha / \mathrm{GPa}^{-1}$ \\
\hline 303.15 & 0.1 & 14.24 & 333.15 & 40 & 11.25 \\
& 10 & 13.66 & & 50 & 10.85 \\
313.15 & 20 & 13.21 & & 60 & 10.51 \\
& 0.1 & 13.92 & & 70 & 10.22 \\
& 10 & 13.27 & 343.15 & 0.1 & 13.35 \\
& 20 & 12.72 & & 10 & 12.67 \\
323.15 & 30 & 12.28 & & 20 & 12.06 \\
& 0.1 & 13.71 & & 30 & 11.53 \\
& 10 & 13.03 & & 40 & 11.06 \\
& 20 & 12.44 & & 50 & 10.64 \\
& 30 & 11.93 & & 60 & 10.27 \\
& 40 & 11.51 & 358.15 & 0.1 & 12.97 \\
& 50 & 11.15 & & 10 & 12.35 \\
& 60 & 10.86 & & 20 & 11.77 \\
& 70 & 10.64 & & 30 & 11.25 \\
& 0.1 & 13.54 & & 40 & 10.79 \\
& 10 & 12.84 & & 50 & 10.37 \\
& 20 & 12.24 & & 60 & 10.00 \\
& 30 & 11.71 & & 70 & 9.66 \\
\hline \hline
\end{tabular}

The parameters used for the vibrating wire sensor are shown in Table S5. The tungsten wire radius has been determined by calibration using toluene data ${ }^{1}$ at 303.15 and $0.1 \mathrm{MPa}$

\section{Table S5}

Parameters of the vibrating wire sensor at the reference temperatures, $T_{\text {ref }}$.

\begin{tabular}{cccc}
\hline \hline & $\mathrm{T}_{\text {ref }} / \mathrm{K}$ & & \\
\hline Tungsten wire data & & & \\
Radius $^{\mathrm{a}}$ & 303.15 & $R_{S} / \mathrm{m}$ & $150.471 \times 10^{-6}$ \\
Density $^{\mathrm{b}}$ & 293.15 & $\rho_{S} /\left(\mathrm{kg} \cdot \mathrm{m}^{-3}\right)$ & 19230 \\
\hline Resonance under vacuum & & & $1.39 \times 10^{-4}$ \\
Internal damping coefficient & & $\Delta_{0}$ & \\
\hline \hline
\end{tabular}

\footnotetext{
${ }^{\mathrm{a}}$ By calibration; ${ }^{\mathrm{b}}$ From literature ${ }^{2} ;{ }^{\mathrm{c}}$ Measured.
} 
1) M. J. Assael, H. M. T. Avelino, N. K. Dalaouti, J. M. N. A. Fareleira, and K. R. Harris, Reference correlation for the viscosity of liquid toluene from 213 to $373 \mathrm{~K}$ at pressures to $250 \mathrm{MPa}$, Int. J. Thermophys., 2002, 22, 789-79.

2) C.M.B.P. Oliveira, Viscosity of Liquid Hydrocarbons at High Pressure, Imperial College of Science Technology and Medicine, London, United Kingdom, 1991. 